\title{
Feasibility of Noninvasive Positive Pressure Ventilation in the Treatment of Oxygen-Dependent COVID-19 Patients in Peru
}

\author{
Paloma M. Cárcamo, ${ }^{1}$ Manuel Laca-Barrera, ${ }^{2}$ Diana Cabanillas-Silva, ${ }^{3}$ Germán Málaga-Rodríguez, ${ }^{4,5}$ Patricia Mallma-Salazar, ${ }^{1}$ \\ David Moore, ${ }^{6}$ Rebecca Shipley, ${ }^{7}$ Mervyn Singer, ${ }^{8}$ and Patricia J. Garcia ${ }^{1 *}$ \\ ${ }^{1}$ School of Public Health, Cayetano Heredia University, Lima, Peru; ${ }^{2}$ Emergency and Critical Care Department, Naval Medical Center, Callao, Peru; \\ ${ }^{3}$ Emergency Department, Hospital de Emergencias Ate Vitarte, Lima, Peru; ${ }^{4}$ Cayetano Heredia Hospital, Lima, Peru; ${ }^{5}$ School of Medicine, Cayetano \\ Heredia University, Lima, Peru; ${ }^{6}$ Faculty of Infectious and Tropical Diseases and TB Centre, London School of Hygiene and Tropical Medicine, \\ London, United Kingdom; ${ }^{7}$ Institute of Healthcare Engineering, University College London, London, United Kingdom; ${ }^{8}$ Bloomsbury Institute of \\ Intensive Care Medicine, University College London, London, United Kingdom
}

\begin{abstract}
Intensive care is expensive, and availability is limited. Low- and middle-income countries in particular have struggled to cope with the large influx of critically ill patients during the COVID-19 pandemic. Noninvasive respiratory support devices delivering continuous positive airways pressure (CPAP) require less resource and staff expertise compared with invasive mechanical ventilators and can be routinely used outside of intensive care units. This study assessed the use of the UCL-Ventura Wayrachi CPAP device in hospitalized patients with COVID-19 in Peru. A secondary analysis of data collected for a feasibility study commissioned by the Peruvian Ministry of Health was conducted. Data were collected from three hospitals, including patient demographics, clinical data, and outcomes. Forty-five patients were enrolled from July 16 to September 1, 2020. Eight patients (18\%) were intolerant of the CPAP mask. Of the remainder, $18(48.7 \%)$ improved and were discharged from hospital after 6 days. Eight (21.6\%) died while on CPAP and $11(29.7 \%)$ were eventually intubated, of whom two died. In total, 27 (60\%) survived to hospital discharge. Participating physicians noted the device was easy to use and provided patient benefit, though voiced concerns about the strain on hospital oxygen supplies. In conclusion, the UCL Ventura Wayrachi CPAP device proved feasible in COVID-19 patients in Peru, and offered a bridging therapy for patients who required a ventilator when none were available.
\end{abstract}

\section{INTRODUCTION}

The first case of COVID-19 illness in Peru was reported on March 6, 2020. ${ }^{1}$ Despite a national lockdown being declared on March $15,{ }^{2}$ the country has been one of the most affected by the pandemic, reaching the highest mortality per capita of any country by August $2020 .^{3}$

An observational study conducted at a national hospital in Peru found that $80 \%$ of patients required high flow, high concentration oxygen support with a median oxyhemoglobin saturation of $87 \%$. However, $90 \%$ of these patients did not get admitted to intensive care, mainly due to lack of bed capacity. ${ }^{4}$ In addition, the necessary ventilators, infusion pumps, sedative and paralyzing drugs, consumables, and human resources required to intubate and mechanically ventilate a patient safely and effectively were also in short supply. Continuous positive airway pressure (CPAP) devices provide noninvasive ventilatory support to hypoxemic patients when high concentration oxygen is inadequate and intensive care unit (ICU) beds or ventilators unavailable. Continuous positive airway pressure requires less resources and can be routinely administered outside the ICU. Less staff expertise is necessary, and less time is required of medical personnel since patients remain awake and often cooperative. ${ }^{5}$ Despite initial concerns regarding the risk of barotrauma and aerosol generation, ${ }^{6}$ CPAP is now routinely and safely used around the world for respiratory support of COVID-19 patients outside the ICU. ${ }^{7}$ Continuous positive airway pressure is also often used as a bridge therapy to intubation or as an alternative ceiling of care for patients in whom invasive mechanical ventilation is deemed inappropriate, with favorable outcomes. ${ }^{8,9} \mathrm{~A}$

*Address correspondence to Patricia J. Garcia, School of Public Health, Cayetano Heredia University, Av. Honorio Delgado 430, San Martín de Porres, Lima, Peru. E-mail: patricia.garcia@upch.pe downside is that commercially available CPAP devices are costly and thus scarce in low- and middle-income countries (LMIC). Additionally, CPAPs require a pressurized oxygen supply and the oxygen flow rate demands may be higher compared with mechanical ventilators.

In response to the urgent need for CPAP devices to treat COVID-19 patients in the United Kingdom in early 2020, engineers and intensivists from University College London (UCL), University College London Hospital (UCLH), and Mercedes AMG High Performance Powertrains (HPP) reverse engineered the Phillips Respironics WhisperFlow, an out-of-patent, previously CE-marked, and purely mechanical CPAP device that can connect directly to a pressurized oxygen supply. Given unprecedented demands on hospital oxygen supplies, the team implemented design changes to the CPAP and breathing circuits, reducing oxygen utilization by $70 \%$. They rapidly prototyped and bench-tested a low-cost device, the UCL Ventura, conducted healthy volunteer and patient evaluations, secured approval from the United Kingdom Medicines and Healthcare Products Regulatory Agency, and distributed 10,000 devices to the United Kingdom National Health Services within a month of idea conception. ${ }^{10}$ This device generates a high-flow oxygen-air mix by entraining environmental air into a pressurized oxygen supply. It has two manual valves that permit adjustment of air flow and the percentage of administered oxygen. It has no moving or electronic parts and does not require an electricity supply. The only required additional parts are inexpensive consumables (mask, tubing, filters, and positive endexpiratory pressure [PEEP] valves), which can be either single used or could be sterilized for patient reuse, and a reusable in-circuit oxygen concentration analyzer. University College London and Mercedes HPP released open-source designs and manufacturing data under free license to bona fide organizations to support the global COVID-19 response, allowing a group of Peruvian engineers to build a local version, named 
the "Wayrachi," which means "wind" in Quechua. However, Peruvian national guidelines for COVID-19 disease only endorsed the use of noninvasive ventilation in individual negative pressure rooms, ${ }^{11}$ which are not available in most public hospitals, and not as initial management ${ }^{12}$ despite the scarcity of ICU beds nationally. ${ }^{13}$

The Peruvian Ministry of Health received a donation of Wayrachi devices and disposable patient circuits including masks, tubing, filters, and PEEP valves to test its use, and to determine whether they should be incorporated into national guidelines. This study thus assessed the feasibility of use of these CPAP devices in hypoxemic hospitalized patients with COVID-19 in Peru.

\section{MATERIALS AND METHODS}

This is a secondary analysis of data collected for a feasibility study commissioned by the Peruvian Ministry of Health. A total of 20 CPAP devices and 40 disposable circuits were distributed to three institutions: Cayetano Heredia Hospital (Lima), Ate Vitarte Emergency Hospital (Lima), and the Naval Medical Center (Callao). Three physicians from participating institutions took part in a day-long virtual training session with experts from UCL and UCLH. At study end, they were invited to a virtual debriefing session regarding the use of the device and issues faced with its implementation.

The following data were collected: patient demographics (sex, age, body mass index), pre-CPAP physiology (respiratory rate and arterial blood gas parameters), inspired oxygen requirement and devices used, complications and tolerance of CPAP, days on CPAP, requirement for mechanical ventilation, and hospital outcome. The device was deployed on hospitalized patients in dedicated non-ICU wards.

All statistical analyses were performed in Stata version 16 (Stata Corp., College Station, TX). Frequencies and percentages are presented for categorical variables. For quantitative variables, means and SDs are provided for normally distributed data, and medians and interquartile ranges otherwise.

This study was approved by the Cayetano Heredia University Institutional Ethics Review Board with inscription code 204291. Data were anonymized prior to statistical analysis.

\section{RESULTS}

A total of 45 patients were enrolled from July 16 to September 1, 2020. Demographics and clinical characteristics are described in Table 1. Most patients were male (66.7\%), between 50 and 59 years (40\%) and overweight or obese (80\%). Six patients were aged $\geq 70$ years. Thirty-eight $(84.4 \%)$ patients were previously receiving highconcentration oxygen via a reservoir mask (15 L/min) and required additional respiratory support and intensive care admission, which were not available. Patients were tachypneic (median rate 38 breaths per minutes), hypoxemic (median oxygen saturation $85 \%$ ), and $88.9 \%$ of patients had thoracic retractions on inspiration.

Eight (18\%) patients did not tolerate the device after minutes of use due to discomfort/pressure (3), feeling of asphyxiation (4), and exhaustion (1). Of the remaining 37, 18 (48.7\%) improved and were discharged after an average of 6 days and a median duration of CPAP treatment of 5 days (interquartile range [IQR]: 4-7). Eight (21.6\%) patients died on CPAP
TABLE 1

Demographic and clinical characteristics of participants prior to starting CPAP $(N=45)$

\begin{tabular}{|c|c|c|}
\hline Demographic characteristics & Frequency & $\%$ \\
\hline \multicolumn{3}{|l|}{ Sex } \\
\hline Female & 15 & 33.3 \\
\hline Male & 30 & 66.7 \\
\hline \multicolumn{3}{|l|}{ Age } \\
\hline 20-39 years & 8 & 17.8 \\
\hline $40-49$ years & 5 & 11.1 \\
\hline $50-59$ years & 18 & 40.0 \\
\hline $60-69$ years & 8 & 17.8 \\
\hline $70-79$ years & 6 & 13.3 \\
\hline \multicolumn{3}{|l|}{ Body mass index category } \\
\hline Normal & 9 & 20.0 \\
\hline Overweight & 21 & 46.7 \\
\hline Obese (I) & 8 & 17.8 \\
\hline Obese (II) & 7 & 15.6 \\
\hline \multicolumn{3}{|l|}{ Clinical characteristics } \\
\hline Respiratory rate, median (IQR) & \multicolumn{2}{|c|}{$38(28-44)$} \\
\hline Days on CPAP, median (IQR) & \multicolumn{2}{|c|}{$4(2-6)$} \\
\hline \multicolumn{3}{|l|}{ Arterial blood gases } \\
\hline $\mathrm{pH}$, mean (standard deviation) & \multirow{3}{*}{\multicolumn{2}{|c|}{$\begin{array}{l}7.42(0.05) \\
33.5(30.1-43) \\
65.6(50-77.9)\end{array}$}} \\
\hline $\mathrm{pCO}_{2}$, median (IQR) & & \\
\hline $\mathrm{pO}_{2}$, median (IQR) & & \\
\hline
\end{tabular}

therapy, whereas 11 (29.7\%) were eventually intubated and mechanically ventilated after a median duration of CPAP treatment of 2 days (IQR: 1-6), when a ventilator became available. Two of these 11 patients died. Twenty-seven (60\%) of the 45 patients started on CPAP survived and were eventually discharged from hospital.

Physician debriefing session. Three physicians participated in the debriefing sessions. They noted the device was easy to use, and no further training was required beyond the initial training session. Use of the CPAP device required a dedicated multipatient ward; one participating institution had negative-pressure rooms, whereas the others only used well-ventilated areas. The required personal protective equipment was similar to what would be required in the ICU during the pandemic, including N95 or KN95 masks, goggles, and coveralls.

In addition to the face mask, tubing, filters, and valves required to use the flow generator, a nipple and clamp were required to ensure a tight fit of the device to the oxygen supply outlets, which was promptly implemented. Locally sourced masks and tubing were initially procured for use with the CPAP device but physicians noted they did not seal properly and increased oxygen utilization. Consequently, they continued to use the recommended consumables, which were sourced from the United Kingdom through the Peruvian Embassy in London, with no further issues. One site sterilized the disposable elements of the system with ethylene gas and noted this did not initially damage the circuit. However, after about five sterilizations, the PEEP valve and mask start deteriorating.

Following removal of the CPAP device to allow patients to eat, drink, or receive nebulization for airway humidification, physicians noted that patients did not tolerate the device as well, and their oxygen saturation did not rise as previously. Thus, physicians opted to maintain patients on the CPAP device semipermanently with use of in-line humidifying filters, with better results. At one site, patients were fed enterally through nasogastric tubes, though this sometimes affected 
the mask seal. At another site, patients were briefly taken off the device to drink formula.

There was widespread concern about the amount of oxygen required for the CPAP devices to function properly, and the strain placed on the already overloaded hospital oxygen infrastructure.

All participating physicians agreed that patients started on CPAP later during their hospitalization did not respond as well as those started earlier.

\section{DISCUSSION}

In our feasibility study, the use of CPAP in hypoxemic COVID-19 patients not coping with oxygen therapy alone enabled $40 \%$ to survive without invasive ventilation and provided bridge therapy in a further $30 \%$ until ICU beds or ventilators became available. Sixty percent were discharged alive. Similar data have been reported from Italy ${ }^{9}$ and the United Kingdom. ${ }^{10}$

Continuous positive airway pressure therapy proved to be a viable alternative for Peru and for patients not suitable for intubation and mechanical ventilation, as evidenced by the six patients aged 70 and above who would not have been routinely offered ICU admission and mechanical ventilation due to resource limitation, long waiting lists for ICU beds, and poor prognosis, despite the lack of standardized national guidelines for ICU prioritization in Peru. Such usage in COVID-19 patients deemed unsuitable for treatment escalation has also been previously described. ${ }^{14}$ This category includes patients who either do not want or are ineligible for invasive ventilation, usually because of comorbidities, frailty, and/or disease severity. In such cases, though the intervention may not always be lifesaving, it can also be an important palliative measure.

Implementing more CPAP devices could greatly increase hospital capacity to care for severely hypoxemic patients, particularly in the context of massive influxes of patients. A French study noted that $40 \%$ of patients requiring ICU care at their institution had to be transferred due to unavailability of ICU beds and ventilators. ${ }^{15}$ They reported initiating CPAP in hypoxemic patients that were unable to secure a ventilator, with $38 \%$ recovering with CPAP alone and $62 \%$ being eventually intubated. A retrospective study from a United Kingdom hospital reported that $64 \%$ of patients who received CPAP avoided intubation altogether, and they were able to avoid exceeding their maximum ICU occupancy. ${ }^{16}$ Another United Kingdom group reported that 13 (58\%) of their cohort of 24 patients avoided mechanical ventilation altogether, with eventual $79 \%$ hospital survival, thereby saving ICU beds and ventilators for even more needy patients. ${ }^{9}$

Peruvian physicians pointed out their concerns about the oxygen demands of high-flow CPAP. The device draws high flows of oxygen ${ }^{17}$ with an estimated requirement between 21 and $47 \mathrm{~L} / \mathrm{min}$ for a hyperventilating, tachypneic COVID19 patient receiving 60-90\% oxygen, as mimicked by healthy volunteers. ${ }^{18}$ Circuit leaks caused by an ill-fitting mask will allow oxygen to escape and thus increase oxygen flow requirements. By comparison, a typical mechanical ventilator use up to $15 \mathrm{~L} /$ min oxygen though automatic leak compensation can increase oxygen use in some devices to over $200 \mathrm{~L} /$ min. ${ }^{19}$ The configuration of the breathing circuit also significantly influences oxygen flow rates. For example, using a single port instead of a twin port mask may increase oxygen flows by $40 \%$. The high demand of oxygen and loss through leaks are important issues to take into consideration and could become important limitations. Outbreaks of COVID-19 have resulted in widespread shortages of oxygen in Peru and many other LMICs. ${ }^{20,21}$ It is imperative that oxygen supplies be verified and secured, that the patient circuit and mask should be fitted properly, and the device turned off when not in use. Additionally, oxygen stewardship protocols should be clearly defined and implemented in hospital wards, and oxygen use should be monitored in real time.

The incompatibility of locally sourced masks and tubing could also pose a challenge. Sterilizing disposable consumables, as was done in one of the participating sites, could be a useful alternative to deal with shortages of supplies. Whether a medical device can be sterilized or not depends on both manufacturer advice and regulatory approvals. Ethylene gas provides an effective way of sterilizing irregularly shaped devices and does not damage most medical grade materials. However, this requires long processing and aerating times as ethylene oxide is toxic, carcinogenic, and flammable, and may pose an environmental risk if not correctly processed within a catalytic cell. ${ }^{22}$ In addition, as mentioned previously, PEEP valves and masks are prone to deterioration after a few cycles of sterilization. Governments and local health agencies should seek to establish protocols for safe sterilization or ensure adequate supplies of disposables.

Our physicians noted that patients commenced on CPAP later during their hospitalization did not respond as well as those started early. A United Kingdom study found CPAP was associated with a significantly lower risk of death but only in patients with hospital stays of 7 days or less. ${ }^{23}$ Several studies have tried to identify clear parameters to determine when intubation is indicated. Described predictors of intubation in COVID-19 patients managed with CPAP include age, elevated lactate dehydrogenase, low-level improvement in the $\mathrm{PaO}_{2} / \mathrm{FiO}_{2}$ ratio after starting CPAP, ${ }^{24}$ decreased oxygenation $\left(\mathrm{SpO}_{2} / \mathrm{FiO}_{2}\right.$ or $\left.\mathrm{PaO}_{2} / \mathrm{FiO}_{2}\right)$ on hospital admission, elevated neutrophil/lymphocyte ratio and an elevated respiratory rate. ${ }^{25}$ None of these risk factors are however absolute. Other forms of noninvasive advanced respiratory support such as bilevel positive airway pressure or high-flow nasal cannula oxygenation might provide similar benefits to hypoxemic patients; however, they are expensive and rarely available in the country. In addition, high-flow nasal cannulas require more oxygen than CPAP (around 40-60 L/min of oxygen), so they might not be a good option in the setting of oxygen shortages. The UCL Ventura is relatively inexpensive and is manufactured locally (as the Wayrachi), which makes it more easily accessible.

Despite the limitations of this small observational study, there is a clear body of evidence that highlights the benefits that CPAP may bring. A simple device such as the UCL Ventura (or Wayrachi in Peru) is less costly and less complex for the nonspecialist to manage than other existing CPAP devices, with no discernible sacrifice in effectiveness. Provided the oxygen supply bottleneck can be overcome, this makes the UCL Ventura a feasible option for countries with limited access to CPAP devices, inadequate ventilator capacity, and/or lack of financial or human resources to access existing CPAP machines. Other countries can easily build their own local version, as was done with the Wayrachi in Peru. This 
device has now obtained approval from the Peruvian Ministry of Health.

In conclusion, use of the UCL Ventura Wayrachi flow generator device in COVID-19 patients in Peru was not only feasible but appears to reduce the need for mechanical ventilation, to offer a bridge therapy while awaiting ventilator availability, and to offer an alternative ceiling of care above standard oxygen therapy for patients in whom mechanical ventilation is not considered appropriate. Issues such as consumables (masks, tubing) and hospital oxygen availability have to be considered to assure effectiveness. Further studies are needed to better identify predictors of CPAP failure in a variety of settings, including LMICs.

Received March 30, 2021. Accepted for publication June 9, 2021.

Published online July 16, 2021.

Acknowledgment: The American Society of Tropical Medicine and Hygiene has waived the Open Access fee for this article due to the ongoing COVID-19 pandemic.

Authors' addresses: Paloma M. Cárcamo, Patricia Mallma-Salazar, and Patricia J. Garcia, School of Public Health, Cayetano Heredia University, Lima, Peru, E-mails: paloma.carcamo.g@gmail.com, patricia. mallma.s@upch.pe, and patricia.garcia@upch.pe. Manuel LacaBarrera, Emergency and Critical Care Department, Naval Medical Center, Callao, Peru, E-mail: manuellaca@hotmail.com. Diana Cabanillas-Silva, Emergency Department, Hospital de Emergencias Ate Vitarte, Lima, Peru, E-mail: diana.cabanillas@upch.pe. Germán Málaga-Rodríguez, Cayetano Heredia Hospital, Lima, Peru, and Cayetano Heredia University, School of Medicine, Lima, Peru, E-mail: german.malaga@upch.pe. David Moore, Faculty of Infectious and Tropical Diseases and TB Centre, London School of Hygiene and Tropical Medicine, London, United Kingdom, E-mail: aavid.moore@ Ishtm.ac.uk. Rebecca Shipley, Institute of Healthcare Engineering, University College London, London, United Kingdom, E-mail: rebecca.shipley@ucl.ac.uk. Mervyn Singer, Bloomsbury Institute of Intensive Care Medicine, University College London, London, London, United Kingdom, E-mail: m.singer@ucl.ac.uk.

This is an open-access article distributed under the terms of the Creative Commons Attribution (CC-BY) License, which permits unrestricted use, distribution, and reproduction in any medium, provided the original author and source are credited.

\section{REFERENCES}

1. Garcia PJ et al., 2020. COVID-19 response in Latin America. Am J Trop Med Hyg 103: 1765-1772.

2. República L, 2020. Coronavirus Perú: en Decreto Supremo Gobierno Declara Estado de Emergencia por 15 Días. Available at: https://larepublica.pe/politica/2020/03/16/coronavirus-perumartin-vizcarra-declara-estado-de-emergencia-nacional-por30-dias/. Accessed January 25, 2021.

3. Peru LDE, 2020. Now It Battles One Of The Worst Coronavirus Outbreaks. Available at: https://www.npr.org/2020/09/08/ 907084962/peru-locked-down-early-now-it-battles-one-of-theworst-coronavirus-outbreaks. Accessed March 8, 2021.

4. Mejía F et al., 2020. Oxygen saturation as a predictor of mortality in hospitalized adult patients with COVID-19 in a public hospital in Lima, Peru. PLoS One 15: e0244171.

5. Mahase E, 2020. COVID-19: Mercedes F1 to provide breathing aid as alternative to ventilator. BMJ 368: $\mathrm{m} 1294$

6. Arulkumaran N, Brealey D, Howell D, Singer M, 2020. Use of noninvasive ventilation for patients with COVID-19: a cause for concern? Lancet Respir Med 8: e45.

7. McEnery T, Gough C, Costello RW, 2020. COVID-19: respiratory support outside the intensive care unit. Lancet Respir Med 8: 538-539.
8. Nicola M, O'Neill N, Sohrabi C, Khan M, Agha M, Agha R, 2020. Evidence based management guideline for the COVID-19 pandemic-review article. Int J Surg 77: 206-216.

9. Nightingale $\mathrm{R}$ et al., 2020. Is continuous positive airway pressure (CPAP) a new standard of care for type 1 respiratory failure in COVID-19 patients? A retrospective observational study of a dedicated COVID-19 CPAP service. BMJ Open Respir Res 7: e000639.

10. Singer M, Shipley R, Baker T, Cowell A, Brealey D, Lomas D, 2020. The UCL Ventura CPAP device for COVID-19. Lancet Respir Med 8: 1076-1078.

11. EsSalud, 2020. Recomendaciones de Manejo Clínico para los Casos de COVID-19. Available at: http://www.essalud.gob.pe/ ietsi/pdfs/guias/RECOMEND_MANEJO_CLINICO_PARA_ LOS_CASOS_DE_COVID_19.pdf. Accessed March 2020.

12. Ministerio de Salud de Perú. Documento Técnico: Manejo de Personas Afectadas por COVID-19 en Áreas de Atención Crítica. Available at: https://cdn.www.gob.pe/uploads/document/file/ 686689/R.M._N_254-2020-MINSA.PDF. Accessed March 8, 2021.

13. Panamericana de la Salud O, 2020. Monitoreo de la Respuesta de Países Sudamericanos frente a la Pandemia de COVID-19. Available at: https://www.paho.org/es/documentos/monitoreorespuesta-paises-sudamericanos-frente-pandemia-covid-19. Accessed February 9, 2021.

14. Bradley P, Nixon J, Wilson J, 2021. Continuous positive airway pressure (CPAP) as a ceiling of care treatment for hypoxemic respiratory failure due to COVID-19. J Intensive Care Soc. Available at: https://journals.sagepub.com/doi/10.1177/175 1143721996538.

15. Alviset $\mathrm{S}$ et al., 2020. Continuous positive airway pressure (CPAP) face-mask ventilation is an easy and cheap option to manage a massive influx of patients presenting acute respiratory failure during the SARS-CoV-2 outbreak: a retrospective cohort study. PLoS One 15: e0240645.

16. Lawton TO, Wilkinson KM, Corp AP, Javid R, MacNally L, McCooe M, Newton E, 2021. Reduced critical care demand with early CPAP and proning in COVID-19 at Bradford: a single centre cohort. J Intensive Care Soc. Available at: https:// journals.sagepub.com/doi/full/10.1177/17511437211018615.

17. Kempley S, Reeves J, Wilkins A, 2021. High oxygen flow rates with the UCL Ventura CPAP device. Lancet Respir Med 9: e35.

18. UCL, 2020. UCL Ventura Mark II Healthy Volunteers Testing. Available at: https://www.ucl.ac.uk/healthcare-engineering/sites/ healthcare-engineering/files/ucl_ventura_mark_ii_healthy_ volunteer_tests_200420.pdf. Accessed March 8, 2021.

19. Singer M, Shipley R, Baker T, Brealey D, Lomas D, 2021. High oxygen flow rates with the UCL Ventura CPAP device-authors' reply. Lancet Resp Med 9: e36.

20. Defensoría del Pueblo CN, 2020. Con la Segunda ola hay Carencia de Oxígeno en Varias Regiones. Available at: https://canaln.pe/ actualidad/defensoria-pueblo-segunda-ola-hay-falta-oxigenovarias-regiones-n431149. Accessed February 9, 2021.

21. AP News, 2021. Medical Oxygen Scarce in Africa, Latin America Amid Virus, 2021. Available at: https://apnews.com/article/ oxygen-crisis-africa-latin-americaeb0d2731a8613c1ae218db7d32a227a6. Accessed March 8, 2021.

22. Rutala WA, Weber DJ, 2015. Disinfection, sterilization, and control of hospital waste. Mandell, Douglas, and Bennett's Principles and Practice of Infectious Diseases Eighth Edition. Elsevier, 3284-3309.

23. Ashish A, Unsworth A, Martindale J, Sundar R, Kavuri K, Sedda L, Farrier M, 2020. CPAP management of COVID-19 respiratory failure: a first quantitative analysis from an inpatient service evaluation. BMJ Open Respir Res 7: e000692.

24. De Vita N, et al., 2021. Predictors of intubation in COVID-19 patients treated with out-of-ICU continuous positive airway pressure. Pulmonology 20: S2531-0437(21)00002-7.

25. Noeman-Ahmed Y, Gokaraju S, Powrie DJ, Amran DA, Sayed IE, Roshdy A, 2020. Predictors of CPAP outcome in hospitalized COVID-19 patients. Respirology 25: 1316-1319. 